\title{
Influence de l'alimentation et de la saison sur les fractions azotées du lait de vache*
}

\author{
par
}

M. JouRnET et B. RÉMOND**

L'intérêt porté aux matières azotées du lait tient d'une part à l'excellente valeur nutritionnelle pour l'homme des protéines qui en constituent la quasi-totalité, et d'autre part au rapport étroit qui lie le rendement fromager à la teneur en matières azotées du lait [44]. Pour le fromager, ce n'est d'ailleurs que la fraction coagulable des protéines, c'est-à-dire la caséine, qui présente de la valeur, bien que l'utilisation croissante de l'ultrafiltration dans la fabrication de certains fromages [42] donne maintenant un intérêt majeur aux protéines solubles (protéines du petit-lait). Dans ce mémoire, nous étudierons l'influence de l'alimentation et de la saison sur la concentration des principales fractions azotées du lait et sur leur proportion relative.

\section{COMPOSITION ET ORIGINE DES MATIERES AZOTEES}

\section{a) Composition}

Les différents constituants azotés, protéiques et non protéiques, du lait ont été répertoriés et décrits dans de nombreuses revues [8, $30,38,53,79]$. Ils sont généralement regroupés en deux grandes fractions : la fraction protéique qui renferme environ 95 p. 100 de l'azote total du lait, et la fraction non protéique (ANP) qui en renferme environ $5 \mathrm{p} .100$. La première fraction se divise à son tour en deux sous-fractions : la caséine et les protéines du lactosérum.

La caséine renferme 76 à 79 p. 100 de l'azote total du lait. Elle est en fait constituée de diverses protéines (caséines $\alpha_{s}, \beta, \chi$ ) dont les

* Extrait du Rapport Principal présenté au 20 Congrès International de Laiterie, Paris, juin 1978.

** Laboratoire de la Production Laitière, I.N.R.A., C.R.Z.V. de Theix, St-GenèsChampanelle - 63110 Beaumont (France). 
proportions relatives et les principales caractéristiques sont maintenant à peu près établies [53]. Les caséines se distinguent plus ou moins catégoriquement des autres protéines du lait par le fait qu'elles coagulent sous l'action de la présure ou d'une acidification du milieu à un $\mathrm{pH}$ voisin de 4,6 .

Les protéines du sérum contiennent 16 à 19 p. 100 de l'azote total du lait. Elles constituent un groupe plus hétérogène que le précédent tant du point de vue de leur origine que de leur composition chimique ou de leur rôle. Quantitativement, les principales protéines sont l' $\alpha$ lactalbumine, la $\beta$-lactoglobuline, la sérum-albumine, et les immunoglobulines.

La fraction azotée non protéique est très hétérogène ; elle comprend de nombreux produits de dégradation du métabolisme de l'animal et de la mamelle, et en particulier de l'urée. L'azote uréique constitue environ la moitié de l'azote non protéique du lait, mais cette valeur est susceptible de larges variations : 33 à 79 p. 100 [37]. En fait, c'est la variation de la teneur en urée qui explique la majeure partie de la variation de la teneur en azote non protéique $[75,76]$.

\section{b) Origine et synthèse des fractions azotées du lait}

La majeure partie des protéines du lait ( 95 p. 100 d'entre elles), et notamment les caséines, l' $\alpha$-lactalbumine et la $\beta$-lactoglobuline, sont synthétisées dans la glande mammaire à partir des acides aminés prélevés par la mamelle dans le plasma sanguin. Le reste des protéines, et en particulier la sérum-albumine et la majorité des immuno-globulines, provient de protéines préformées dans le sang [35].

Les deux principales sources d'énergie pour le métabolisme de la mamelle sont l'acétate et le glucose prélevés dans le sang. La synthèse de protéines dans la glande mammaire est limitée par la quantité d'acides aminés et d'énergie disponibles [70]. Un apport supplémentaire d'énergie accroît :

1. La quantité d'acides aminés disponible pour la synthèse des protéines du lait, soit à cause de l'accroissement de la quantité de protéines microbiennes synthétisées dans le rumen puis absorbées dans l'intestin [29], soit par un rôle d'épargne du supplément de glucose ou d'acide propionique absorbé, sur les acides aminés ; des infusions de protéines dans le duodénum ont provoqué une augmentation de la quantité de protéines synthétisée et de leur taux dańs le lait [cf. 12] ;

2. La quantité de nutriments énergétiques absorbée (acides gras volatils, glucose). L'acide propionique pourrait d'ailleurs jouer un rôle spécifique ; comparativement à l'acide acétique, son instillation dans le rumen provoque une augmentation du taux protéique du lait [58]. L'acide propionique stimule beaucoup plus la synthèse des protéines que celle du lactose (qui détermine la quantité d'eau excrétée), alors que l'acide acétique accroît davantage la sécrétion de lactose 
et de matières grasses que celle de protéines. L'acide propionique pourrait agir comme précurseur du glucose ou par son action insulinosécrétrice : activation des synthèses protéiques et diminution du catabolisme azoté [18].

Les composés azotés non protéiques (ANP) du lait sont des déchets du métabolisme de la mamelle et, ou, proviennent du sang. Différents auteurs ont montré une diminution du taux d'urée sanguine entre l'entrée et la sortie de la mamelle, et Journet et al. [32] ont établi une corrélation étroite $(r=0,91)$ entre la teneur en urée du sang et celle du lait. En outre, une proportion variable de l'urée du lait provient d'une désamination interne d'acides aminés prélevés par la mamelle $[43,75]$.

\section{VARIATIONS COMPAREES DE LA CONCENTRATION DES FRACTIONS PROTEIQUES ET NON PROTEIQUES}

Les différences de teneurs en matières azotées entre des laits de mélange sont dues pour la majeure partie à la fraction protéique (tab. 1). L'amplitude de variation de la concentration de la fraction azotée non protéique ne représente guère, en moyenne, plus de 10 p. 100 de celle des matières azotées totales, alors que celle de la fraction caséine en représente près de 85 p. 100. Ce n'est qu'en valeur relative que la concentration de la fraction azotée non protéique varie beaucoup plus que celle de la fraction protéique.

Les différences de taux des fractions azotées observées selon les régimes, les saisons, les troupeaux, sont probablement dues pour une large part, aux facteurs du milieu, alimentaire et saisonnier. Dans une étude récente réalisée à l'INRA, dans laquelle les types de vache et d'alimentation étaient variés, nous avons pu montrer que le régime alimentaire avait un effet plus faible sur la teneur en caséine ou en protéines solubles que le niveau de production laitière ou le stade de lactation, mais plus élevé que la race ou l'âge des animaux (tab. 2). En outre, le régime alimentaire avait, sur la teneur du lait en ANP, un effet plus élevé que ceux des facteurs caractérisant les animaux.

\section{NIVEAU DES APPORTS ENERGETIQUES}

L'accroissement des apports énergétiques provoque un enrichissement du lait en extrait sec dégraissé qui est dû en presque totalité aux matières azotées [59]. Pour des apports variant de -20 à +40 p. 100 des besoins énergétiques, la teneur en matières azotées du lait s'accroît sans modification appréciable de la proportion relative de la caséine : 77,8 p. 100 et 77,6 p. 100 pour les niveaux extrê- 
TABLEAU 1

Variabilités comparées des teneurs en $\mathrm{N}$ total, $\mathrm{N}$ caséique et $\mathrm{N}$ non protéique de laits de mélange, entre régions, saisons ou troupeaux différents

\begin{tabular}{|c|c|c|c|c|c|c|}
\hline \multirow{2}{*}{ Auteurs } & \multirow{2}{*}{ Facteurs de variation } & \multicolumn{3}{|c|}{$\begin{array}{l}\text { Ecart entre les valeurs extrêmes } \\
\text { (mg d'N/100 g de lait) }\end{array}$} & \multirow{2}{*}{$\begin{array}{c}3 / 1 \\
(\text { p. } 100)\end{array}$} & \multirow{2}{*}{$\begin{array}{c}2 / 1 \\
(\mathrm{p} .100)\end{array}$} \\
\hline & & $\begin{array}{l}\mathrm{N} \text { total } \\
\text { (1) }\end{array}$ & $\begin{array}{l}\text { N caséine } \\
(2)\end{array}$ & $\begin{array}{c}\mathrm{N} \text { non } \\
\text { protéique (3) }\end{array}$ & & \\
\hline $\begin{array}{l}\text { NICKERSON (1960) } \\
\text { Californie (U.S.A.) }\end{array}$ & 6 régions & 77 & 63 & 3 & 4 & 82 \\
\hline $\begin{array}{l}\text { HARLAND (1955) } \\
\text { Ensemble des U.S.A. }\end{array}$ & 81 laits de troupeaux & 288 & 253 & 19 & 7 & 88 \\
\hline $\begin{array}{l}\text { Mc DowELL (1972) } \\
\text { Nouvelle-Zélande }\end{array}$ & $\begin{array}{l}433 \text { laits de troupeaux frisons } \\
159 \text { laits de troupeaux jersey }\end{array}$ & $\begin{array}{l}169 \\
251\end{array}$ & & $\begin{array}{l}30 \\
29\end{array}$ & $\begin{array}{l}18 \\
12\end{array}$ & - \\
\hline $\begin{array}{l}\text { VIGNON (1972) } \\
\text { Est de la France }\end{array}$ & 4 troupeaux (mai-juin) & 20 & & 8 & 40 & - \\
\hline $\begin{array}{l}\text { SHAHANI et SOMMER (1951) } \\
\text { Wisconsin (U.S.A.) }\end{array}$ & 14 laits de mélange & 91 & 77 & 11 & 12 & 85 \\
\hline $\begin{array}{l}\text { NICKERSON (1960) } \\
\text { Californie (U.S.A.) }\end{array}$ & 4 saisons & 33 & 27 & 0 & 0 & 82 \\
\hline $\begin{array}{l}\text { Burton (1967) } \\
\text { Sud de l'Angleterre }\end{array}$ & 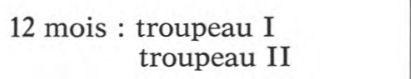 & $\begin{array}{l}73 \\
88\end{array}$ & $\begin{array}{l}54 \\
78\end{array}$ & $\begin{array}{r}13 \\
9\end{array}$ & $\begin{array}{l}18 \\
10\end{array}$ & $\begin{array}{l}74 \\
89\end{array}$ \\
\hline
\end{tabular}


TABLEAU 2

Influence des principaux facteurs de variation des fractions protéiques et non protéiques du lait : pourcentage de variance expliqué (1)

\begin{tabular}{|c|c|c|c|c|c|c|}
\hline Fractions azotées & Facteurs de variations & Race & $\begin{array}{l}\text { Numéro } \\
\text { de } \\
\text { lactation }\end{array}$ & $\begin{array}{l}\text { Niveau de } \\
\text { production } \\
\text { laitière }\end{array}$ & $\begin{array}{l}\text { Stade } \\
\text { de } \\
\text { lactation }\end{array}$ & Régime \\
\hline $\begin{array}{c}\text { g } \mathrm{N} \times 6,39 \\
\text { par } \mathrm{kg} \text { de } \\
\text { lait }\end{array}$ & $\begin{array}{l}\text { Matières azotées totales } \\
\text { Caséine } \\
\text { Protéines solubles } \\
\text { Matières azotées non protéiques }\end{array}$ & $\begin{array}{r}6 \\
6 \\
3 \\
11\end{array}$ & $\begin{array}{l}0 \\
1 \\
1 \\
1\end{array}$ & $\begin{array}{l}37 \\
31 \\
26 \\
15\end{array}$ & $\begin{array}{l}41 \\
33 \\
28 \\
20\end{array}$ & $\begin{array}{l}22 \\
17 \\
12 \\
37\end{array}$ \\
\hline $\begin{array}{l}\text { p. } 100 \text { des matières } \\
\text { azotées totales }\end{array}$ & $\begin{array}{l}\text { Caséine } \\
\text { Matières azotées non protéiques }\end{array}$ & $\begin{array}{l}3 \\
8\end{array}$ & $\begin{array}{l}5 \\
2\end{array}$ & $\begin{array}{l}4 \\
0\end{array}$ & $\begin{array}{l}4 \\
2\end{array}$ & $\begin{array}{r}5 \\
20\end{array}$ \\
\hline
\end{tabular}

(1) Analyse de variance à 1 seul facteur sur 109 échantillons de lait individuels journaliers, 5 races, 3 numéros de lactation (1, 2, 3 et plus), 7 niveaux de production laitière, 7 régimes (d'après A. Le Dore, 1977). 


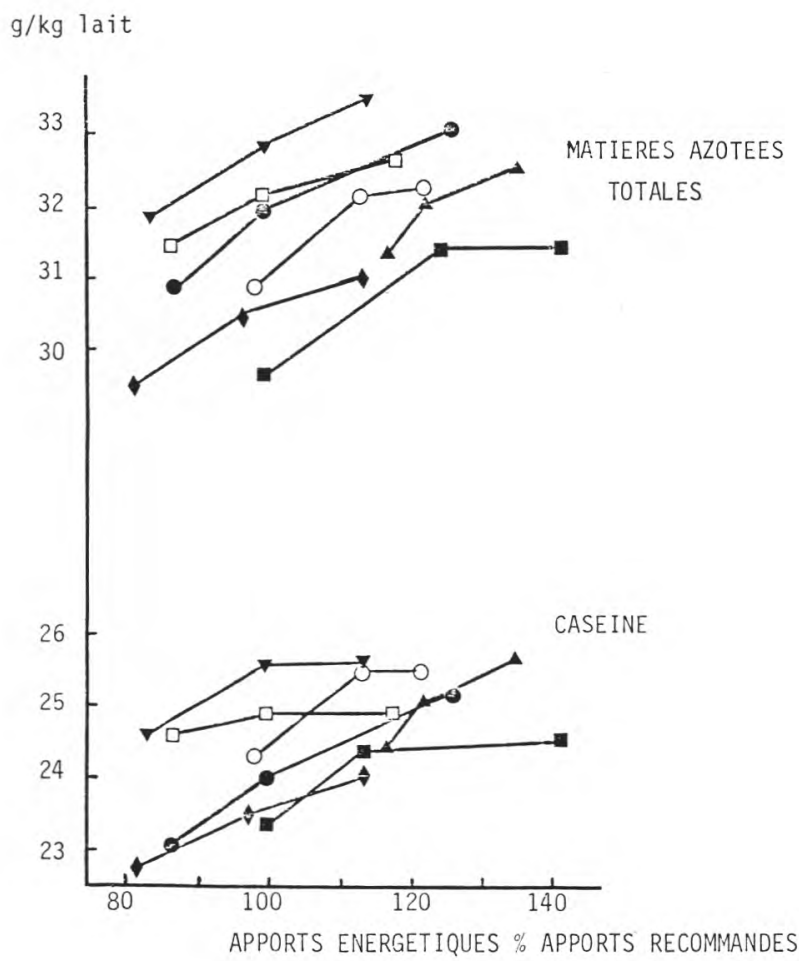

fig. 1

Influence du niveau des apports énergétiques de la ration sur le taux des matières azotées totales et de caséine du lait

○ Holmes et al., 1956.

- Holmes et al., 1960.

- Castle et al., 1958.

A Castle et al., 1961.
- Rook et Line, 1961.

$\nabla$ Nordfeldt et Ruudvere, 1963.

Nordfeldt et Claesson, 1964.

mes d'apport énergétique (fig. 1). L'accroissement du taux de caséine dans le lait obéit à la loi des rendements décroissants : il a été trois fois plus faible entre le niveau moyen et le niveau élevé $(0,24 \mathrm{~g}$ p. 1000) qu'entre le niveau bas et le niveau moyen $(0,81 \mathrm{~g} \mathrm{p} .1000)$.

Ces résultats sont en accord avec ceux d'une étude réalisée en France à l'INRA, dans laquelle 1029 échantillons individuels de lait ont été analysés. Une variation de 10 p. 100 du niveau des apports énergétiques (1,1 UF par vache et par jour, en moyenne) accompagnée d'une variation dans le même sens des apports azotés, a provoqué un accroissement de 0,4 g p. 1000 du taux des matières azotées sans modification 
significative de la proportion de caséine [36]. En Finlande, Ettala [17] a observé un accroissement du taux des protéines dans le lait de $0,70 \mathrm{~g}$ p. 1000 par unité fourragère.

En cas de diminution très importante (de moitié) de niveau des apports alimentaires, accompagnée éventuellement d'un changement dans la composition des rations, la diminution du taux des matières azotées (3 à $4 \mathrm{~g}$ p. 1000) a été entièrement due à la caséine dont la proportion relative a donc diminué. Cette diminution n'a cependant pas dépassé trois points p. 100 [66].

Les variations de proportion relative des différentes fractions protéiques du lait ont été peu étudiées. Il semble cependant qu'un accroissement de la proportion de concentré dans la ration n'accroisse que les quantités de protéines synthétisées dans la mamelle et donc leur proportion relative [80].

Une diminution de la concentration de l'ANP dans le lait et de la proportion de l'ANP dans $1^{\prime} \mathrm{N}$ total du lait consécutivement à une augmentation de l'apport énergétique (par l'alimentation ou l'instillation d'acides gras volatils dans le rumen) a été observée par de nombreux auteurs $[19,59,75]$. Pour les mêmes apports azotés, une augmentation de 50 p. 100 des apports énergétiques fait diminuer la teneur en ANP du lait de $8 \mathrm{mg}$ d'azote par $100 \mathrm{ml}$ et la proportion de l'ANP dans l'N total de 1,5 point p.100. Cette plus faible teneur en ANP du lait quand le niveau énergétique augmente explique en majeure partie pourquoi la proportion de caséine dans l'azote total est plus élevée avec les régimes à concentration énergétique élevée.

\section{NIVEAU D'APPORT AZOTE ET NATURE DES SOURCES AZOTEES}

A même niveau d'apport énergétique, le niveau des apports azotés influence très peu la teneur en matières azotées du lait [24]. Le léger accroissement de la teneur en MAT du lait de 0,12 g p. 1000 observé par Rook et Line [60] consécutif au passage des apports azotés alimentaires de 70 p. 100 à 165 p. 100 des besoins n'a porté que sur la fraction azotée non protéique. Des liaisons étroites entre la teneur en azote de la ration et la teneur en azote non protéique du lait ont été observées par différents auteurs $[62,78]$. En fait, la teneur en azote non protéique du lait est encore davantage liée au rapport : apports énergétiques/apports azotés ; quand ce rapport passe de 1 à 1,5 , la teneur en ANP du lait diminue de $9 \mathrm{mg} / 100 \mathrm{~g}$ [19].

Les variations de la teneur du lait en ANP portent presque exclusivement sur l'azote uréique comme l'ont bien montré Ide et al. [26] qui ont observé une liaison très étroite $(\mathrm{r}=0,979)$ entre le taux azoté de la ration et le taux d'urée du lait. Cependant, l'amplitude de variation de la teneur en ANP du lait reste faible : elle augmente d'environ 
$10 \mathrm{mg}$ d'azote par $100 \mathrm{ml}$ de lait quand la teneur en MAT de la ration passe de 12 à 18 p. 100 [32]. Bien que faibles, ces variations sont cependant suffisantes pour modifier légèrement la proportion relative des fractions azotées du lait. Dans l'étude effectuée sur les troupeaux expérimentaux de l'INRA, la proportion de caséine a diminué de 0,7 point p. 100 lorsque les apports azotés se sont accrus de 10 p. 100 , à même niveau d'apports énergétiques [36].

Deux raisons peuvent expliquer l'absence d'effet du niveau des apports azotés alimentaires sur le taux protéique du lait :

1. La quantité d'acides aminés absorbée dans l'intestin augmente généralement peu consécutivement à un accroissement de l'apport de matières azotées dans la ration, car celles-ci sont en grande partie (50 à 90 p. 100) catabolisées dans le rumen.

2. L'accroissement de la quantité d'acides aminés absorbée dans l'intestin entraîne à la fois un accroissement de la quantité de lait et de protéines produites, de sorte que le taux protéique varie peu. Ainsi, consécutivement à un apport de tourteaux de soja et de colza tannés, nous n'avons pas observé d'augmentation significative de la teneur du lait en matières azotées bien que les quantités de matières azotées sécrétées se soient accrues [73]. Cependant, lorsque des quantités importantes de caséine ont été instillées à l'entrée du duodénum, des accroissements à la fois de la quantité et du taux de caséine du lait ont été observés, ainsi que du taux d'ANP [12]. Inversement le taux d'ANP du lait est bas en début de lactation consécutivement à des faibles apports azotés d'origine alimentaire et microbienne [52, 73].

En revanche, dans le cas de pâturages fortement fertilisés (plus de $350 \mathrm{~kg}$ d'azote/ha/an) et donc très riches en azote soluble très facilement dégradable en ammoniaque dans le rumen, des teneurs élevées du lait en ANP, supérieures à $300 \mathrm{mg}$ par $\mathrm{kg}$, ont été observées $[3,4,50]$. Elles se sont parfois accompagnées d'une diminution de l'acidité titrable, d'une augmentation du temps de coagulation du lait et d'une diminution de l'élasticité du coagulum [5], ce qui semble montrer que des apports azotés excessifs peuvent modifier la composition en matières azotées du lait. Les conséquences que ce déséquilibre alimentaire pourrait avoir sur les qualités technologiques du lait nécessitent cependant d'être confirmées.

Le remplacement dans la ration d'une partie non excessive (20 à 30 p. 100) de l'azote protéique des tourteaux par de l'azote non protéique, lorsque les rations avaient une concentration énergétique suffisante, n'ont occasionné de changements ni de la teneur en matières azotées totales, ni de la proportion des différentes fractions azotées du lait, et en particulier de l'ANP [27, 40, 54, 78]. Le remplacement de la totalité de l'azote protéique par de l'azote non protéique (uréique ou ammoniacal), associé à une répartition des apports azotés au cours de la journée, n'a pas entraîné d'accroissement de la teneur en ANP du lait dans les essais de Virtanen [77] et Syvaoja et al. [67]. 
D'ailleurs lorsque les apports de glucides très digestibles, utilisés dans ces rations synthétiques ont été synchronisés avec l'apport d'azote non protéique, de très faibles pourcentages d'azote non protéique dans le lait ont été observés [77].

En revanche, lorsque l'addition d'urée à la ration conduit à un apport excessif d'azote fermentescible par rapport à la matière organique digestible dans le rumen, c'est-à-dire lorsque l'apport de PDIN est supérieur à l'apport de PDIE dans le nouveau système d'expression des besoins azotés [cf. 29], le taux d'ANP dans le lait augmente [64]. C'est également ce qui se produit lorsque les matières azotées des autres composants de la ration sont trop dégradables [63].

\section{COMPOSITION DE LA RATION}

\section{Proportion de fourrages et d'aliment concentré}

L'accroissement de l'apport énergétique de la ration s'effectue généralement par un apport supplémentaire de concentré. Il en résulte une modification de la production des acides gras volatils dans le rumen : la production des acides propionique et butyrique s'accroît alors que celle de l'acide acétique diminue ou reste inchangée. Ces modifications peuvent entraîner un accroissement plus important de la production de protéines dans le lait que de celle de lactose et de la quantité d'eau et donc une augmentation du taux azoté du lait.

La liaison entre le taux des protéines du lait synthétisées dans la mamelle et la proportion des acides gras volatils dans le rumen a cependant été contestée par Yousef et al. [80] ; ces auteurs ont en effet enregistré un taux élevé de ces protéines dans le lait aussi bien avec des rations à proportion élevée de céréales induisant une proportion élevée d'acide propionique et faible d'acide acétique dans le rumen, qu'avec les mêmes rations additionnées de correcteurs qui ont rétabli des proportions normales d'acides gras volatils. Mais ces rations riches en céréales, en maïs surtout, peuvent aussi agir en augmentant la quantité d'amidon qui passe dans l'intestin. Or, le glucose issu de l'hydrolyse de l'amidon a un effet semblable à celui de l'acide propionique. De sorte que si ces rations ont bien accru le taux protéique du lait dans certains essais [21, 41, 71] les mécanismes en cause ne sont pas parfaitement connus.

\section{Composition de la ration fourragère hivernale}

Parmi les différents fourrages qui constituent les rations hivernales, l'ensilage d'herbe est celui qui occasionne le plus souvent les taux de matières azotées dans le lait les plus faibles. Cet effet dépressif a souvent été attribué au plus faible niveau des apports énergétiques consécutifs à un bas niveau d'ingestion [cf. 33]. L'addition de céréales 
ou de betteraves à la ration permet d'ailleurs de relever le taux azoté du lait [cf. 2 et 33].

Le niveau des apports énergétiques ne semble cependant pas seul en cause. Nordfeldt et Claesson [47] ont en effet montré qu'à même niveau d'apport énergétique le taux de matières azotées totales et de caséine était d'autant plus faible que la proportion d'ensilage d'herbe était plus élevée (entre 25 p. 100 et 75 p. 100 de la MS de la ration) et la proportion d'aliment concentré plus faible. Dans l'essai réalisé à I'INRA, le taux de matières azotées totales du lait a été de 1,2 à $1,4 \mathrm{~g}$ p. 1000 plus faible avec les rations à base d'ensilage d'herbe qu'avec celles à base de foin ou d'ensilage de maïs (36 et tab. 3). La différence a porté davantage sur les protéines solubles que sur la caséine, de sorte que la proportion de caséine dans les matières azotées totales a été plus élevée avec l'ensilage d'herbe qu'avec le foin. La proportion des matières azotées non protéiques dans les MAT, et des matières azotées uréiques dans les matières azotées non protéiques ont été les plus faibles avec les rations à base d'ensilage de maïs. Comparativement à l'ensilage d'herbe, l'association d'ensilage d'herbe et de maîs a permis un accroissement considérable de la teneur en matières azotées du lait qui a porté à la fois sur la caséine et les protéines solubles, sans modification de la teneur en ANP. Dans l'essai les ensilages d'herbe étaient hachés finement et additionnés d'acide formique ; la qualité de la plupart d'entre eux était bonne malgré leur faible teneur en matière sèche (18 à 22 p. 100). Cet effet dépressif de l'ensilage d'herbe sur le taux de protéines du lait est peut-être dû à son effet stimulant sur la quantité de lait produite, attribué à une ingestion élevée d'acide acétique. C'est l'hypothèse qu'ont émise Nordfeldt et Claesson [47] après avoir constaté, comme Rook et Balch [58], qu'une infusion d'acide acétique dans le rumen tendait à provoquer une diminution du taux des protéines du lait.

Les betteraves ont généralement un effet favorable sur le taux des protéines du lait $[10,11,49]$. Leur effet propre est difficile à séparer d'un effet du niveau des apports énergétiques ; cependant, l'augmentation de la proportion relative de l'acide propionique et surtout butyrique dans les acides gras volatils totaux du rumen [72] est par elle-même favorable à un accroissement des synthèses protéiques dans la mamelle.

\section{Pâturage}

La mise à l'herbe au printemps provoque le plus souvent un accroissement de la teneur en matières azotées du lait qui peut atteindre 2 à 3 g p. 1000 [cf. 2, 33, 57] et qui se réalise progressivement en 2 à 3 semaines [15]. Cet accroissement porte sur la caséine et les protéines solubles $[28,55,56]$. Il faut l'attribuer à l'élévation du niveau des apports énergétiques qui a généralement lieu entre la fin de la période hivernale et le début de la période de pâturage, et 
TABLEAU 3

Influence du régime alimentaire sur les teneurs et les proportions de différentes fractions azotées du lait

\begin{tabular}{|c|c|c|c|c|c|c|c|c|}
\hline \multirow{3}{*}{$\begin{array}{c}\text { Fractions } \\
\text { azotées }\end{array}$} & \multirow{3}{*}{$\begin{array}{c}\text { Régime } \\
\text { alimentaire }\end{array}$} & \multicolumn{5}{|c|}{ Ration de base } & \multirow{3}{*}{$\begin{array}{l}\text { Valeur } \\
\text { moyenne }\end{array}$} & \multirow{3}{*}{$\begin{array}{l}\text { Plus } \\
\text { petite } \\
\text { différence } \\
\text { significa- } \\
\text { tive }\end{array}$} \\
\hline & & \multirow{2}{*}{ Foin } & \multirow{2}{*}{$\begin{array}{l}\text { Ensilage } \\
\text { d'herbe }\end{array}$} & \multirow{2}{*}{$\begin{array}{l}\text { Ensilage } \\
\text { de maîs }\end{array}$} & \multicolumn{2}{|c|}{ Pâturage } & & \\
\hline & & & & & Printemps & Automne & & \\
\hline & Matières azotées totales & $-0,34$ & $-1,57$ & $-0,20$ & $+0,62$ & $+1,49$ & 35,05 & 0,59 \\
\hline $\mathrm{g}$ & Caséine & $-0,29$ & $-1,00$ & $+0,02$ & $+0,27$ & $+1,00$ & 27,12 & 0,46 \\
\hline $\mathrm{N} \times 6,39 / \mathrm{kg}$ & Protéines solubles & $+0,02$ & $-0,33$ & $+0,02$ & $+0,07$ & $+0,21$ & 6,05 & 0,15 \\
\hline lait & Matières azotées non protéiques & $-0,07$ & $-0,24$ & $-0,24$ & $+0,27$ & $+0,28$ & 1,87 & 0,06 \\
\hline & Matière azotée uréique & $-0,10$ & $-0,18$ & $-0,30$ & $+0,29$ & $+0,30$ & 0,92 & 0,06 \\
\hline $\begin{array}{l}\text { p. } 100 \\
\text { matières }\end{array}$ & Caséine & $-0,11$ & $+0,64$ & $+0,49$ & $-0,58$ & $-0,44$ & 77,39 & 0,32 \\
\hline $\begin{array}{l}\text { azotées } \\
\text { totales }\end{array}$ & Matières azotées non protéiques & $-0,10$ & $-0,43$ & $-0,71$ & $+0,68$ & $+0,57$ & 5,36 & 0,15 \\
\hline
\end{tabular}

Résultats de l'analyse de variance effectuée sur 559 échantillons de lait individuels, après élimination des effets dus aux différences de race (Frisonne, Holstein, Frisonne $\times$ Holstein, Montbéliarde, Normande), de stade de lactation (quatre périodes), et de niveau de production (d'après A. Le Dore, 1977). 
peut-être aussi à un changement de composition de la ration et de la proportion des produits terminaux de la digestion dans le rumen. Différents auteurs [1,6] ont en effet observé un accroissement de la teneur en acides gras volatils dans le jus de rumen, et de la proportion d'acide propionique. Le taux de protéines du lait ne s'est d'ailleurs pas accru lorsque le niveau d'apport énergétique du régime hivernal précédant la mise à l'herbe était élevé $[55,56,78]$.

De nombreux auteurs ont observé une augmentation rapide et temporaire de la teneur en ANP du lait de l'ordre de 5 à $8 \mathrm{mg}$ d'N/100 $\mathrm{ml}[16,45,55,64,69,76]$. On doit l'attribuer à la consommation d'une herbe jeune, riche en matières azotées qui provoque une élévation du taux d'urée plasmatique et consécutivement du taux d'urée du lait ; la variation du taux d'urée du lait explique en totalité les variations du taux d'ANP du lait à cette période [76].

Les teneurs du lait les plus élevées aussi bien en protéines qu'en matières azotées non protéiques sont observées au cours du pâturage d'automne. Ainsi, la différence de plus de 2 g p. 1000 dans le taux des matières azotées du lait entre un pâturage d'automne et les régimes hivernaux [36] a été due pour 55 p. 100 à la caséine, 12 p. 100 aux protéines solubles et 33 p. 100 à l'azote non protéique. De sorte que la proportion plus faible de caséine dans les matières azotées totales observée en automne ( 1 point dans cette étude) est due presque entièrement aux teneurs élevées en azote non protéique du lait ; mais la proportion de caséine dans les protéines totales reste inchangée. A cette période, la proportion de l'azote non protéique dans l'azote total a représenté jusqu'à 7,5 p. 100 de l'azote total du lait [68]. Ces valeurs élevées doivent être attribuées à la très grande richesse de l'herbe en azote, et principalement en azote soluble. Les teneurs les plus élevées $(38 \mathrm{mg} / 100 \mathrm{ml})$ en azote non protéique dans le lait ont été observées avec des plantes telles que la luzerne, pour lesquelles le rapport protéines solubles/glucides solubles + amidon est élevé ; et les valeurs les plus faibles ( 5 à $7 \mathrm{mg}$ ) avec le maïs pour lequel ce rapport est faible [3].

\section{EFFET DE LA SAISON}

Dans les pays tempérés où, généralement, les vaches sont au pâturage l'été et passent l'hiver en stabulation, la concentration des protéines dans le lait passe par 2 minima (en fin d'hiver et au milieu de l'été), et 2 maxima (à la mise à l'herbe et à la fin de la période de pâturage) (fig. 2). Cette évolution est due à la fois à la caséine et aux protéines solubles dont les concentrations évoluent de façon semblable au cours de l'année. L'écart entre les concentrations mensuelles maximales et minimales de caséine observées sur des laits de troupeau ou de laiterie a été en moyenne de 2,0 g p. 1000 pour différents auteurs (cf. tab. 4) avec des extrêmes de 0,6 et 4,7 g p. 1000. Pour les protéines 

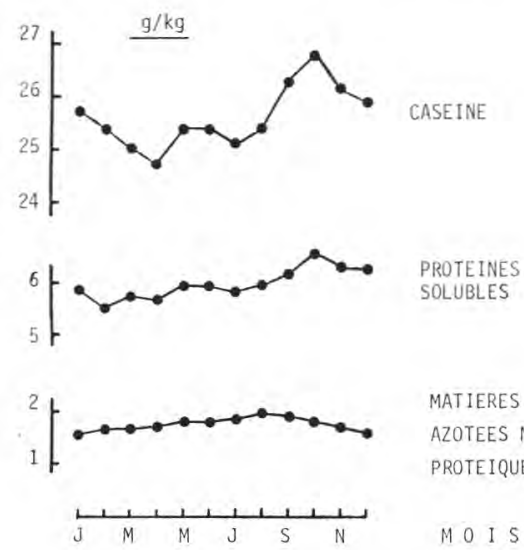

MATIERES

AZOTEES NON PROTEIQUES

MO I S

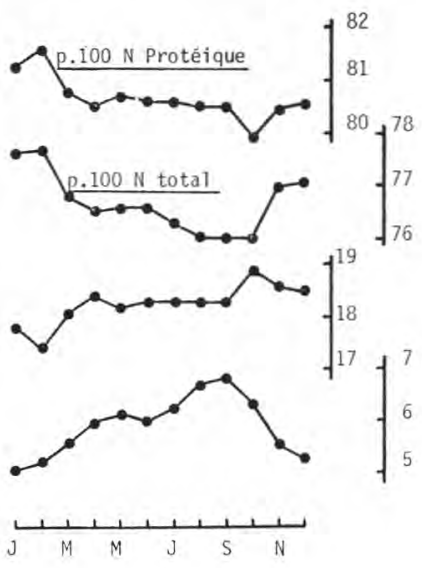

fig. 2

Evolution saisonnière des teneurs et des proportions de la caséine, des protéines solubles et des matières azotées non protéiques dans le lait. (Moyenne des valeurs observées par Davies, 1935 ; Reinart et Nesbitt, 1961 ; Ter Horst, 1963 ; Harding et Royal, 1974 ; Joost et al., 1974).

solubles, l'écart moyen a été de 0,66 g p. 1000 (mêmes auteurs ; cf. tab. 4) avec des valeurs extrêmes de 0,3 et $1,5 \mathrm{~g}$ p. 1000.

La teneur en azote non protéique est en général la plus faible pendant la période hivernale. Elle augmente à la mise à l'herbe mais elle n'atteint sa valeur maximale qu'à la fin de l'été et au début de l'automne (août, septembre). Mahieu et al. [39] ont observé une évolution tout à fait semblable du taux d'urée dans des laits de grand mélange provenant des différentes régions de France et analysés mensuellement. L'écart entre les concentrations maximales et minimales d'ANP a été, en moyenne, faible en valeur absolue (65 mg d'N par litre) mais très variable ( 7 à $97 \mathrm{mg}$ ) et élevé en valeur relative (en moyenne, 23 p. 100 de la moyenne) (tab. 4).

L'écart entre les concentrations mensuelles extrêmes de caséine a été en moyenne 3 fois plus élevé que celui concernant les protéines solubles, et 4,8 fois plus élevé que celui concernant les matières azotées non protéiques (ANP $\times 6,38)$ (tab. 4). Compte tenu de l'évolution semblable de la caséine, des protéines solubles et, dans une moindre mesure, de l'azote non protéique, la proportion de la caséine dans les matières azotées totales varie relativement peu au cours de l'année : l'écart entre les proportions mensuelles maximales et minimales a été en moyenne de 1,8 point p. 100 (tab. 5). Quand on rapporte la concentration de la caséine aux seules protéines, la différence entre les proportions maximales et minimales n'est plus que de 1,4 point. Les proportions de caséine (dans les MAT ou les protéines) les 


\section{TABLEAU 4}

Teneurs maximales et minimales du lait en caséine, en protéines solubles et en azote non protéique au cours de l'année

\begin{tabular}{|c|c|c|c|c|c|c|c|}
\hline \multirow{4}{*}{ Références } & \multirow{4}{*}{$\begin{array}{l}\text { Caractéristiques des études } \\
\text { (pays, échantillonnage, durée, nombre } \\
\text { d'échantillons) }\end{array}$} & \multicolumn{6}{|c|}{ Concentration des fractions azotées } \\
\hline & & \multicolumn{2}{|c|}{ Caséine } & \multicolumn{2}{|c|}{ Protéines solubles } & \multirow{2}{*}{\multicolumn{2}{|c|}{\begin{tabular}{|l} 
Azote non protéique \\
mg pour $1 \mathrm{~kg}$ ou $11^{*}$
\end{tabular}}} \\
\hline & & \multicolumn{4}{|c|}{ g pour $1 \mathrm{~kg}$ ou $11^{*}$} & & \\
\hline & & Max. & Min. & Max. & Min. & Max. & Min. \\
\hline Davies, 1935 & Angleterre : 1 troupeau, 2 ans, 96 échantillons & $\stackrel{25,8}{N}$ & $\begin{array}{c}23,3 \\
\text { Ao }\end{array}$ & $\stackrel{6,0}{S}$ & $\begin{array}{l}5,3 \\
\text { Ao }\end{array}$ & $\begin{array}{c}322 \\
\mathrm{D}\end{array}$ & $\begin{array}{l}263 \\
\mathrm{Ms}\end{array}$ \\
\hline $\begin{array}{l}\text { REINART et } \\
\text { NESBITT, } 1961\end{array}$ & $\begin{array}{l}\text { Canada : } 3 \text { troupeaux Holstein, } 2 \text { ans, } 72 \text { échan- } \\
\text { tillons }\end{array}$ & $\begin{array}{c}26,4^{*} \\
\mathrm{Ja}\end{array}$ & $\begin{array}{c}25,4^{*} \\
\mathrm{Jt}\end{array}$ & $\begin{array}{l}6,0^{*} \\
0\end{array}$ & $\stackrel{5,2^{*}}{\mathrm{~F}}$ & $\begin{array}{l}357^{*} \\
\text { Ao }\end{array}$ & $\begin{array}{l}260^{*} \\
\mathrm{~F}\end{array}$ \\
\hline $\begin{array}{l}\text { HARLAND et al., } \\
1955\end{array}$ & $\begin{array}{l}\text { Etats-Unis : Laiteries de } 10 \text { Etats, } 3 \text { périodes : } \\
\mathrm{F}, \mathrm{Ms}, \mathrm{Ju}, \mathrm{O}, 81 \text { échantillons }\end{array}$ & - & - & $\begin{array}{l}6,9 \\
\text { Jn }\end{array}$ & $\begin{array}{c}6,1 \\
\text { F-Ms }\end{array}$ & - & - \\
\hline NiCKERSON, 1960 & $\begin{array}{l}\text { Etats-Unis (Californie) : } 6 \text { laiteries, } 20 \text { mois, } 4 \\
\text { périodes, } 258 \text { échantillons }\end{array}$ & $\begin{array}{l}27,4 \\
\text { N-D-Ja }\end{array}$ & $\stackrel{25,7}{\text { Mi-Jn-Jt }}$ & $\begin{array}{l}\text { 6,2 } \\
\text { N-D-Ja, } \\
\text { Ao-S-O }\end{array}$ & $\stackrel{5,8}{\text { Mi-Jn-Jt }}$ & $\begin{array}{l}315 \\
\text { N-D-Ja }\end{array}$ & $\begin{array}{l}308 \\
\text { Mi-Jn-Jt }\end{array}$ \\
\hline TER HoRst, 1963 & $\begin{array}{l}\text { Pays-Bas : Laiteries de Frise, } 8 \text { ans, } 300 \text { échan- } \\
\text { tillons }\end{array}$ & $\begin{array}{l}27,8 * \\
0\end{array}$ & $\begin{array}{l}23,1^{*} \\
\mathrm{Av}\end{array}$ & $\begin{array}{l}7,5^{*} \\
\mathrm{O}\end{array}$ & $\begin{array}{l}6,0^{*} \\
\mathrm{Av}\end{array}$ & $\begin{array}{l}260^{*} \\
F\end{array}$ & $\begin{array}{l}210^{*} \\
\mathrm{O}, \mathrm{N}, \mathrm{D}, \mathrm{Ja}\end{array}$ \\
\hline Joost et al., 1973 & Suède : 15 laiteries, 6 ans, 1000 échantillons & 27,3 & $\begin{array}{l}25,6 \\
\mathrm{Mi}\end{array}$ & - & - & - & - \\
\hline $\begin{array}{l}\text { HARDING et } \\
\text { ROYAL, } 1974\end{array}$ & $\begin{array}{l}\text { Grande-Bretagne : } 17 \text { laiteries, } 23 \text { ans, } 3500 \\
\text { échantillons }\end{array}$ & $\begin{array}{c}26,2 \\
0\end{array}$ & $\begin{array}{c}23,8 \\
\mathrm{Ms}-\mathrm{Av}\end{array}$ & $\begin{array}{l}6,1 \\
\text { S-O }\end{array}$ & $\begin{array}{l}5,5 \\
\text { F-Ms-Av }\end{array}$ & $\begin{array}{c}345 \\
\mathrm{~S}\end{array}$ & $\begin{array}{c}251 \\
\mathrm{Ja}\end{array}$ \\
\hline SASVARI, 1971 & $\begin{array}{l}\text { Hongrie : Laits individuels, } 2 \text { périodes : été et } \\
\text { hiver, } 1300 \text { échantillons }\end{array}$ & $\begin{array}{l}28,3 \\
\text { Hiv. }\end{array}$ & $\begin{array}{l}27,7 \\
\text { été }\end{array}$ & $\begin{array}{l}8,1 \\
\text { hiv. }\end{array}$ & $\begin{array}{l}7,8 \\
\text { été }\end{array}$ & & \\
\hline LE DORE, 1977 & $\begin{array}{l}\text { France : Laits individuels de } 4 \text { troupeaux : } 3 \\
\text { périodes : F, Av-Mi, O, } 1200 \text { échantillons }\end{array}$ & $\begin{array}{c}28,2 \\
0\end{array}$ & $\begin{array}{c}26,8 \\
\mathrm{~F}, \mathrm{Av}-\mathrm{Mi}\end{array}$ & $\stackrel{6,4}{0}$ & $\begin{array}{l}\text { 6,0 } \\
\text { F, Av-Mi }\end{array}$ & $\begin{array}{c}329 \\
0\end{array}$ & $\begin{array}{c}247 \\
\text { F }\end{array}$ \\
\hline
\end{tabular}

Ja : janvier; F : février; Ms : mars; Av: avril ; Mi : mai ; Jn : juin ; Jt: juillet ; Ao : août ; S : septembre ; O : octobre ; $\mathrm{N}$ : novembre; $\mathrm{D}$ : décembre. 
Proportions relatives maximales et minimales au cours de l'année de la caséine, des protéines solubles et des matières azotées non protéiques dans le lait

\begin{tabular}{|c|c|c|c|c|c|c|c|c|c|}
\hline \multirow{3}{*}{ Références } & \multirow{3}{*}{$\begin{array}{l}\text { Caractéristiques des études } \\
\text { (pays, échantillonnage, durée, } \\
\text { nombre d'échantillons) }\end{array}$} & \multicolumn{6}{|c|}{ p. 100 matières azotées totales } & \multirow{2}{*}{\multicolumn{2}{|c|}{$\begin{array}{l}\text { p. } 100 \text { protéines } \\
\text { Caséine }\end{array}$}} \\
\hline & & \multicolumn{2}{|c|}{ Caséine } & \multicolumn{2}{|c|}{ Protéines solubles } & \multicolumn{2}{|c|}{$\begin{array}{c}\text { Matières azotées } \\
\text { non protéiques }\end{array}$} & & \\
\hline & & Max. & Min. & Max. & Min. & Max. & Min. & Max. & Min. \\
\hline DAVIES, 1935 & Cf. tab. 4 & $\begin{array}{c}77,4 \\
\mathrm{Ja}\end{array}$ & $\begin{array}{c}76,2 \\
\mathrm{~F}, \mathrm{Av}, \mathrm{S}, \mathrm{O}\end{array}$ & $\begin{array}{l}18,5 \\
\mathrm{Av}\end{array}$ & $\begin{array}{c}16,9 \\
N\end{array}$ & $\begin{array}{l}6,5 \\
\text { Ao }\end{array}$ & $\begin{array}{l}5,2 \\
\mathrm{Ja}\end{array}$ & $\stackrel{82,0}{\mathrm{~N}}$ & $\begin{array}{l}80,4 \\
\mathrm{Av}\end{array}$ \\
\hline $\begin{array}{ll}\text { REINART } & \text { et } \\
\text { NESBITt, } & 1961\end{array}$ & Cf. tah. 4 & $\stackrel{79,1}{F}$ & $\begin{array}{c}76,5 \\
\mathrm{O}\end{array}$ & $\stackrel{17,7}{\mathrm{~N}}$ & $\begin{array}{l}15,8 \\
\text { Ms }\end{array}$ & $\begin{array}{l}6,9 \\
\text { Ao }\end{array}$ & $\stackrel{5,1}{F}$ & $\begin{array}{c}83,3 \\
\mathrm{~F}, \mathrm{Ms}\end{array}$ & $\begin{array}{l}81,3 \\
\mathrm{O}, \mathrm{N}, \mathrm{D}\end{array}$ \\
\hline $\begin{array}{l}\text { DE KONING et } \\
\text { al., } 1974\end{array}$ & $\begin{array}{l}\text { Pays-Bas : } 1 \text { troupeau, } 1 \\
\text { an, } 21 \text { échantillons }\end{array}$ & $\begin{array}{c}75,6 \\
\mathrm{Jn}\end{array}$ & $\begin{array}{c}73,4 \\
O\end{array}$ & $\begin{array}{c}20,6 \\
0\end{array}$ & $\begin{array}{c}18,2 \\
\mathrm{Jt}\end{array}$ & $\begin{array}{l}7,0 \\
\mathrm{Jt}\end{array}$ & $\begin{array}{l}5,6 \\
\mathrm{~F}, \mathrm{Ms}\end{array}$ & $\begin{array}{c}80,5 \\
\mathrm{Jt}\end{array}$ & $\stackrel{78,1}{0}$ \\
\hline $\begin{array}{l}\text { HARLAND et al., } \\
1955\end{array}$ & Cf. tab. 4 & - & - & - & - & - & - & $\begin{array}{l}81,8 \\
\text { F-Ms }\end{array}$ & $\begin{array}{c}79,3 \\
\mathrm{Ju}\end{array}$ \\
\hline NICKERSON, 1960 & Cf. tab. 4 & $\begin{array}{c}77,0 \\
\text { N-D-Ja }\end{array}$ & $\begin{array}{c}76,5 \\
\text { Ao-S-O }\end{array}$ & $\begin{array}{l}17,8 \\
\text { Mi-Jn-Jt } \\
\text { N-D-Ja }\end{array}$ & $\begin{array}{l}17,4 \\
\text { Mi-Jn-Jt }\end{array}$ & $\begin{array}{c}5,9 \\
\text { N-D-Ja }\end{array}$ & $\begin{array}{c}5,6 \\
\text { N-D-Ja }\end{array}$ & $\begin{array}{c}81,6 \\
\text { Ao-D-Ja }\end{array}$ & $\begin{array}{c}81,1 \\
\text { Ao-S-O }\end{array}$ \\
\hline TER Horst, 1963 & Cf. tab. 4 & $\begin{array}{l}76,9 \\
\mathrm{Mi}\end{array}$ & $\begin{array}{l}75,2 \\
\text { Ms }\end{array}$ & $\begin{array}{c}20,5 \\
0\end{array}$ & $\stackrel{18,4}{F}$ & $\stackrel{5,0}{F}$ & $\stackrel{3,7}{0}$ & $\stackrel{80,6}{F}$ & $\begin{array}{c}78,7 \\
0\end{array}$ \\
\hline $\begin{array}{l}\text { GRAPPIN et } \\
\text { JEUNET, } 1973\end{array}$ & $\begin{array}{l}\text { France : } 1 \text { laiterie, } 5 \text { ans, } \\
60 \text { échantillons }\end{array}$ & $\begin{array}{c}79,4 \\
\mathrm{D}\end{array}$ & $\begin{array}{l}78,1 \\
S, O\end{array}$ & - & - & $\begin{array}{c}5,6 \\
\mathrm{Jt}\end{array}$ & $\begin{array}{c}4,2 \\
\mathrm{D}\end{array}$ & - & - \\
\hline Joost et al., 1973 & Cf. tab. 4 & $\begin{array}{c}79,0 \\
\mathrm{D}\end{array}$ & $\stackrel{77,6}{S}$ & & & & & & \\
\hline $\begin{array}{l}\text { SZIJARTO et al., } \\
1973\end{array}$ & $\begin{array}{l}\text { Canada : } 24 \text { laiteries, } 1 \text { an, } \\
874 \text { échantillons }\end{array}$ & $\stackrel{77,2}{F}$ & $\begin{array}{l}73,1 \\
\text { S-O }\end{array}$ & $\begin{array}{c}19,9 \\
0\end{array}$ & $\stackrel{17,8}{\mathrm{~F}}$ & $\stackrel{7,6}{S}$ & $\begin{array}{c}4,9 \\
\mathrm{Ja}\end{array}$ & $\stackrel{81,3}{F}$ & $\begin{array}{c}78,6 \\
0\end{array}$ \\
\hline $\begin{array}{l}\text { HARDING et } \\
\text { ROYAL, } 1974\end{array}$ & Cf. tab. 4 & $\begin{array}{c}77,1 \\
\mathrm{Ja}\end{array}$ & $\begin{array}{c}75,5 \\
\text { Ao }\end{array}$ & $\begin{array}{c}18,1 \\
\text { Ao }\end{array}$ & $\begin{array}{c}17,6 \\
\text { Ao }\end{array}$ & $\stackrel{6,5}{S}$ & $\begin{array}{l}5,1 \\
\mathrm{Ja}\end{array}$ & $\stackrel{81,3}{F}$ & $\begin{array}{l}80,7 \\
\text { Ao }\end{array}$ \\
\hline SASVARI, 1971 & Cf. tab. 4 & - & - & - & - & - & - & $\begin{array}{l}78,0 \\
\text { été }\end{array}$ & $\begin{array}{c}77,7 \\
\text { hiver }\end{array}$ \\
\hline LE DoRE, 1977 & Cf. tab. 4 & $\begin{array}{c}78,0 \\
F\end{array}$ & $\begin{array}{c}76,9 \\
0\end{array}$ & $\stackrel{17,4}{F}$ & $\begin{array}{l}17,2 \\
\mathrm{Mi}\end{array}$ & $\begin{array}{c}5,8 \\
0\end{array}$ & $\stackrel{4,6}{F}$ & $\begin{array}{l}81,7 \\
\text { F, Av-Mi }\end{array}$ & $\begin{array}{c}81,5 \\
0\end{array}$ \\
\hline
\end{tabular}


plus élevées ont généralement été observées en hiver, et les proportions les plus faibles, pendant la saison de pâturage, et en particulier en automne.

Les variations de concentration et de proportion relative des différentes fractions azotées au cours de la saison ne sont pas dues qu'aux variations des caractéristiques climatiques et saisonnières (température, hygrométrie, durée du jour...), mais à la conjonction de différents facteurs parmi lesquels, outre la saison, le stade de lactation des vaches et l'alimentation sont vraisemblablement les plus importants. Il est d'ailleurs à noter que, dans les résultats de Nickerson [46] obtenus en Californie où, généralement, les vêlages sont répartis au cours de l'année et l'alimentation varie peu, la concentration de la caséine et des protéines solubles varie peu au cours de l'année (écarts entre valeurs mensuelles extrêmes de respectivement 2 g p. 1000 et 0,4 g p. 1000) ; il en est de même de la concentration de l'ANP (écart de $7 \mathrm{mg} \mathrm{p.1000)}$ ainsi que de la proportion de caséine (écart de 0,5 point p. 100). Après élimination, par traitement statistiques, de l'effet du stade de lactation, Sasvari [61] et Le Dore [36] ont observé de faibles variations de la proportion de caséine dans les protéines totales (tab. 5).

Les modifications dans l'alimentation des animaux sont responsables de la variation de la teneur en ANP du lait, et vraisemblablement aussi des variations rapides des teneurs en caséine et en protéines solubles à certaines périodes (mise à l'herbe, fin de la saison de pâturage). Mais les variations de grande amplitude du taux des protéines synthétisées dans la mamelle correspondent à un effet saisonnier indépendant de l'alimentation; le minimum en été et le maximum en hiver des taux de caséine [46] et de protéines totales [7], de même que du taux butyreux, d'ailleurs [7], sont à mettre en relation directe avec la variation de la durée du jour [cf. 14].

\section{CONCLUSION}

L'alimentation a une certaine influence sur le taux d ss différentes fractions protéiques, qui est plus faible cependant que celle des caractéristiques individuelles (race, stade de lactation, niveau de production laitière). Deux facteurs alimentaires ont un effet prépondérant :

1. Le niveau des apports énergétiques qui influe sur le taux des protéines synthétisées dans la mamelle ; ce facteur explique une partie importante des variations de teneurs observées entre les différents types de régimes et entre les saisons.

2. Le niveau des apports azotés qui influe surtout sur le taux de la fraction azotée non protéique et presque exclusivement sur la teneur en urée. Les variations de la teneur du lait en azote non protéique sont 
fortes en valeur relative comparativement à celles des fractions protéiques, mais faibles en valeur absolue. Cependant, ces variations qui ont lieu indépendamment de celles des fractions protéiques, expliquent une part importante des variations de la proportion de la caséine dans les matières azotées totales. La concentration des deux fractions protéiques, caséine et protéines solubles, varie le plus souvent dans lé même sens, et leur rapport varie assez peu. Les propriétés technologiques différentes de ces deux fractions nécessitent cependant que des études plus nombreuses sur leurs variations relatives soient entreprises.

\section{S u m m a r y}

INFLUENCE OF FEEDING AND SEASON ON THE NITROGENOUS FRACTIONS

IN COW'S MILK

We reviewed the bibliography concerning the feeding and seasonal effects on the concentration of the principal nitrogenous fractions (casein, soluble proteins, non-protein nitrogen) in cow's milk and on their relative proportions.

The differences in the concentration of crude protein (total $\mathrm{N} \times 6.38$ ) between bulk milks are mainly ( 85 p. 100 on the average) due to differences in the concentration of proteins (table 1).

Variation in the plane of energy nutrition modifies the concentration of crude protein in milk, in the same way; the relative proportion of casein is little affected (fig. 1).

Variation in the plane of protein nutrition modifies the nonprotein nitrogen content in milk in the same way; this change is essentially due to variation in urea content.

Basal diets have particular effects on the concentration of nitrogenous fractions in milk, but have little influence on relative proportion of casein in the crude protein (table 3).

Season has an effect on the protein content in milk, which is highest when the daylight duration is short, and lowest when the daylight duration is long (fig. 2 - tabl. 5 and 6). The relative proportion of the casein is little affected by season. 


\section{Bibliographie}

[1] Annison (E. F.), Lewis (D.), Lindsay (D. B.) (1959). - J. Agric. Sci., 53, 34-41.

[2] Auriol (P.), JARrige (R.) (1962). - Fédération Internationale de Laiterie, Bulletin annuel, $2^{\mathrm{e}}$ partie.

[3] Bakanov (V. N.), Ovsisher (B. R.), Bondareva (N. I.), Mamaev (V. A.), FilipPOV (V. F.) (1976), - In: Nutr. Abstr. Rev., Series B, 1977, 47 B, 174.

[4] Barabanshchikov (N. V.) (1972). - Dairy Sci. Abstr., 1974, 36, 375.

[5] Barabas (J.) (1976). - In: Dairy Sci. Abstr., 1977, 39, 378.

[6] Bath (I. H.), Rook (J. A. F.) (1965). - J. Agric. Sci., 64, 67-75.

[7] BRUhn (J. C.), Franke (A. A.) (1977). - J. Dairy Sci., 60, 696-700.

[8] BRunner (R. J.) (1977). - In: Food Proteins, édité par J.R. Whitaker et S. R. Tannenbaum, p. 175-208. Avi Publishing Company, Inc., Westport, Connecticut (U.S.A.).

[9] Burton (H.) (1967). - J. Dairy Res., 34, 137-143.

[10] Castle (M. E.), Mac Lusky (D. S), Waite (R.), Watson (J. N.) (1958). - J. Dairy Res., 25, 365-372.

[11] Castle (M. E.), Drysdale (A. D.), Waite (R.) (1961). - J. Dairy Res., 28, 67-74.

[12] CLARK (J. H.) (1975). - In: Protein nutritional quality of foods and feeds. Part 2, p. 261-304. Edité par Mendel Friedman et Marcel Dekker, Inc., New York.

[13] Davies (W. L.) (1935). - J. Dairy Res., 6, 363-368.

[14] Decaen (C.), Journet (M.) (1966). - Ann. Zootech., 15, 259-277.

[15] Demarouilly (C.), Journet (M.) (1962). - $16^{\mathrm{e}}$ Congrès Intern. Laiterie, Copenhague, Section I, 1, 33-49.

[16] Devuyst (A.), Vanbelle (M.), Paquet (R.), Arnould (R.), Vervack (W.), Moreels (A.) (1964). - Agricultura, Louvain, 12, 205-222.

[17] Ettala (E.) (1976). - Ann. Agric. Fenn., 15, 182-195.

[18] Evans (E.), Buchanan-Smith (J. G.), Mac Leod (G. K.), Stone (J. B.) (1975). J. Dairy Sci., 58, 672-677.

[19] Gordon (F. J.), Forbes (T. J.) (1970). - J. Dairy Res., 37, 481-491.

[20] Grappin (R.), Jeunet (R.) (1973). - Rapport présenté aux journées du Cercle International des Chercheurs Laitiers, tenues à Poligny, les 15, 16, 17 mai 1973.

[21] Haenlein (G. F. W.) (1972). - Dissert. Abstr. Intern., B, 33, 1869-1870.

[22] Harding (F.), Royal (L.) (1974). - Dairy Industries, octobre, 372-378.

[23] Harland (H. A.), Coulter (S. T.), Jenness (R.) (1955). - J. Dairy Sci., 38, 858-869.

[24] Holmes (W.), Waite (R.), Mac Lusky (D. S.), Watson (J. N.) (1956). J. Dairy Res., 23, 1-12.

[25] Holmes (W.), Arnold (G. W.), Provan (A. L.) (1960). - J. Dairy Res., 27, 191-204.

[26] Ide (Y.), Ogura (Y.), Yonemura (T.) (1966). - In: Dairy Sci. Abstr., 1967, 29, 451.

[27] Iwata (H.) (1958). - XV Intern. Dairy Congr., 1-A-9, 54-58.

[28] JARRIGE (R.) (1953). - Ann. Zootech., 2, 33-53. 
[29] JARrige (R.), Journet (M.), Vérité (R.) (1978). - In : L'alimentation des ruminants, p. 89-128. Ed. INRA Publications (route de Saint-Cyr) - 78000 Versailles.

[30] JENNess (R.) (1974). - In: Lactation, vol. 3, edited by B. L. Larson and V. R. Smith. Academic Press.

[31] Joost (K.), Soderkvist (A.), Overstrom (H.) (1973). - Svenska Mejeriernas Riksförening. U.P.A. Meddelande $\mathrm{nr} 94$.

[32] Journet (M.), Vérité (R.), Vignon (B.) (1975). - Le Lait, 543-544, 212-223.

[33] Kirchgessner (M.), Friesecke (H.), Koch (G.) (1967), - Crosby Lockwood and Son Ltd, London.

[34] de Koning (P. J.), Koops (J.), Van Rooijen (P. J.) (1974). - Neth. Milk Dairy J., 27, 186-202.

[35] LARSON (B. L.), Jorgensen (G. N.) (1974). - In: Lactation, vol. 2, edited by B. L. Larson and V. R. Smith, Academic Press.

[36] LE DoRe (A.) (1977). - Thèse Ingénieur-Docteur, Université des Sciences et Techniques du Languedoc.

[37] Mc Doweli (A. K. R.) (1972). - J. Dairy Res., 39, 27-33.

[38] Mac Kenzie (H. A.) (1967). - In: Advances in Protein Chemistry, vol. 22, 56-221, Academic Press.

[39] Mahieu (H.), Luquet (F. M.), Mouillet (L.) (1976). - Le Lait, 559-560, 657-698.

[40] Mariani (P.), Catalano (A.) (1975), - Rev. Zoot. e Vet., 3, 253-271.

[41] Mariani (P.), Russo (V.), Armibaldi (S.), Nanni (M.) (1975). - Riv. Zoot. $e$ Vet., 5, 399-413.

[42] MauboIs (J. L.) (1978). - 20 Congrès International de Laiterie, Paris.

[43] Mepham (T. B.), Linzell (J. L.) (1967). - Nature, 214, 507-508.

[44] Mocouot (G.), Ricordeau (G.), Auriol (P.) (1963). - Ann. Zootech., 12, 53-66.

[45] Neseni (R.), KoRPRich (H.) (1948). - Milchwissenschaft, 3, 183-186.

[46] Nickerson (T. A.) (1960). - J. Dairy Sci., 43, 598-606.

[47] NordFeldt (S.), Claesson (O.) (1964). - Lantbrukshögsk. Ann., 30, 517-545.

[48] Nordfeldt (S.), RuUdvere (A.) (1963). - Lantbrukshögsk. Ann., 29, 345-393.

[49] ORth (A.), KaufMann (W.) (1964). - Milchwissenschaft, 19, 164-167.

[50] Palfi (F. Y.), Vudmaska (V. Y.), Kulik (M. S.), Lesiv (S. N.) (1976) + - In: Nutr. Abstr. Rev., Series B, 1977, 47 B, 810.

[51] Reinart (A.), Nesbitt (J. M.) (1961). - The composition of milk in Manitoba. Department of Dairy Science, University of Manitoba.

[52] Remond (B.), Journet (M.) (1978). - Ann. Zootech., 27, 139-158.

[53] Ribadeau-Dumas (B.) (1978). - 20 Congrès International de Laiterie, Paris.

[54] Robertson (N. H.), Dixon (A.) (1969). - S. Afr. J. Dairy Techn., 1, 39-43.

[55] Rook (J. A. F.), Rowland (S. J.) (1958). - XV Intern. Dairy Congress (London), 1-B-20, 204-209,

[56] Rook (J. A. F.), Line (C.), Rowland (S. J.) (1960), - J. Dairy Res., 27, 427-433.

[57] Rook (J. A. F.) (1961). - Dairy Sci. Abstr., 23, 251-258.

[58] Rook (J. A. F.), BaLCH (C. C.) (1961). - Brit. J. Nutr., 15, 361-369.

[59] Rook (J. A. F.), LINE (C.) (1961). - Brit. J. Nutr., 15, 109-119.

[60] Rook (J. A. F.), LiNe (C.) (1962). - XVI Intern. Dairy Congress, Copenhagen, A, 57.

[61] Sasvari (Z.) (1961). - Acta Agronomica Scientiarum Hungaricae, 20, 73-78.

[62] Schalitscheff (Y.) (1966). - XVIIth Intern. Dairy Congress, Munich, Section $\mathrm{A}, 1,75-80$. 
[63] Schingoethe (D. J.), Voelker (H. H.), Beardsley (G. L.), Parsons (J. G.) (1976). - J. Dairy Sci., 59, 894-901.

[64] Senft (B.), Klobasa (F.) (1969). - Milchwissenschaft, 24, 713-717.

[65] Shahani (K. M.), Sommer (H. H.) (1951). - J. Dairy Sci., 34, 1010-1013.

[66] Stobbs (T. H.), Brett (D. J.) (1974). - Aust. J. Agric. Res., 25, 657-666.

[67] Syvadja (E. L.), Ettala (T.), Kreula (M.) (1975). - In: Dairy Sci. Abstr., 1975, 37, 793.

[68] Szijarto (L.), Biggs (D. A.), Irvine (D. M.) (1973). - J. Dairy Sci., 56, 45-51.

[69] Ter Horst (M. G.) (1963). — Neth. Milk Dairy J., 17, 162-175.

[70] Thomas (P. C.) (1975). - World Review of Animal production, 11 (4), 33-44.

[71] VASIC (J.), ZeRemsKi (D.), Zivkovic (Z.) (1974). - Veterinaria, 23, 201-209.

[72] Vérité (R.), Journet (M.) (1973). - Ann. Zootech., 22, 219-235.

[73] Vérité (R.), Journet (M.) (1977). - Ann. Zootech., 26, 183-205.

[74] Vignon (B.) (1972). - Résultats non publiés.

[75] Vignon (B.) (1976). - Thèse Doctorat ès Sciences. Université de Nancy I.

[76] Vignon (B.), Laurent (F.), Journet (M.) (1978). - Ann. Zootech., 27, 303-315.

[77] Virtanen (A. I.) (1967). - Neth. Milk and Dairy J., 21, 223-244.

[78] Waite (R.), Castle (M. E.), Watson (J. N.), Drysdale (A. D.) (1968). - J. Dairy Res., 35, 191-202.

[79] Whitney (R. Mcl.), Brunner (J. R.), Ebner (K. E.), Farrell (H. M.), JosephSON (R. V.), MORR (C. V.), Swaisgood (H. E.) (1976). - J. Dairy Sci., 59, 795-815.

[80] Yousef (I. M.), Huber (J. T.), Emery (R. S.) (1970). - J. Dairy Sci., 53, 734-739. 